\title{
Designing and manufacturing aspherical polystyrene lenses for the terahertz region
}

\author{
A. Shevchik-Shekera ${ }^{1,}$, V. Zabudsky'1, A. Golenkov¹, S. Dvoretsky² \\ ${ }^{1} V$. Lashkaryov Institute of Semiconductor Physics, NAS of Ukraine, Kyiv, Ukraine \\ *E-mail: annashsh82@gmail.com
}

${ }^{2}$ A.V. Rzhanov Institute of Semiconductor Physics, RAS, pr. Lavrentieva, 13, Novosibirsk, Russian Federation

\begin{abstract}
We report on the development of lenses for terahertz vision systems from their design up to manufacturing. Fused deposition modeling was used for assisting the 3D printing manufacturing process. HIP (High Impact Polystyrene) used for printing has high transmittance in the terahertz region and low deformation in the manufacturing process. The comparative analysis of experimental parameters data and the designed ones showed good agreement for the aspherical lenses manufactured. Application of the lenses manufactured to get imaging of some objects at $140 \mathrm{GHz}$ radiation with 32 elements linear detector array has been demonstrated.
\end{abstract}

Keywords: 3D printing, HIPS, THz lenses, THz region.

doi: https://doi.org/10.15407/spqeo21.01.083

PACS 42.15.Eq

Manuscript received 21.02.18; revised version received 14.03.18; accepted for publication 29.03.18; published online 29.03.18.

\section{Introduction}

Today there exists an ongoing interest in terahertz $(\mathrm{THz})$ technologies. The present-day technological development in the $\mathrm{THz}$ range opens new areas of applications in different domains, namely: medicine, security, food control, etc. These technologies continue their advance mainly in terms of detectors and sources [1-3]. Also, one of the important tasks in $\mathrm{THz}$ instrumentation development is to improve quasi-optic elements for various active $\mathrm{THz}$ vision systems.

The majority of $\mathrm{THz}$ systems (e.g., pulsed $\mathrm{THz}$ systems with photoconductive antennas, continuous wave (CW) photomixer systems) operates basically in the $\mathrm{THz}$ spectral range $v \sim 0.2 \ldots 4 \mathrm{THz}$, their output power lies in the range $P<1 \mu \mathrm{W}$. That's why, as a rule, single detectors are used, and images are acquired using slow raster scanning systems, which takes several minutes to complete relatively small area image, because of the source has a low power. Real-time or close to real-time imaging can be attained using high-power sources such as THz QCLs (Quantum Cascade Lasers) or some others (e.g., the based on, IMPATT (impact ionization avalanche transit-time) diodes, Gunn oscillators, BWOs (Backward Wave Oscillators), etc. [4, 5]) and using multielement $\mathrm{THz}$ detectors disposed at the plane of irradiation with a relatively large area.
Here, aspheric lenses to focus $\mathrm{THz}$ radiation into linear multielement detector array were designed and manufactured on the base of HIPS (High Impact Polystyrene) by 3D printing process. That enabled to get close to real-time imaging of some objects with a multielement linear detector array by using a relatively powerful IMPATT diode and clinotron (variant of the conventional BWO) sources.

\section{Designing and manufacturing the terahertz aspherical lenses}

The report about aspherical lenses for $\mathrm{THz}$ imaging can be found in [6]. There were demonstrated various approaches to the aspherical lens design. One of the most common methods for production is mechanical formation of lenses. These lenses usually have good quality, but they are expensive and hard to produce.

In our early papers [7, 8], we proposed lenses for $\mathrm{THz}$ systems based on the refractive aspherical ones. Plastic PTFE (Polytetrafluoroethylene) with ultra-high molecular weight and refractive index of 1.43 was selected as material for lenses. The lenses were processed using a mechanical/polishing lathe, which resulted in a surface roughness less than $30 \mu \mathrm{m} \quad(\sim \lambda / 10)$. The diffraction-limited system with four identical planoconvex aspherical lenses was obtained. All these lenses were tested using the special stand. For all the lenses, the 
focal distance was $70 \mathrm{~mm}$ and diameter equal to $60 \mathrm{~mm}$. The calculated and experimental data have close values $\left(D_{\text {Airy-calc }}=5.6 \mathrm{~mm}, \quad D_{\text {Airy-meas }} \approx 8 \mathrm{~mm}\right)$, respectively. Therefore, this type of lenses can be used for THz/subTHz imaging systems [5, 6].

In this work, we used another approach for manufacturing lenses related to the $3 \mathrm{D}$ printing capabilities, which reduce manufacturing time, increases cost-efficiency, and allows establishing the full cycle of creating aspherical $\mathrm{THz}$ lenses in the one workplace.

The widespread introduction of 3D printing additive technologies, including the method of fused deposition modeling (FDM), allows creating $\mathrm{THz}$ optics elements for scientific and technical applications [1-4].

FDM is an additive manufacturing technology that builds parts up layer-by-layer by heating and extruding thermoplastic filament. This technology is clean, low cost and office-friendly.

The FDM printing resolution is of the order of $60 \ldots 100 \mu \mathrm{m}$ and depends on the used material. Therefore, this method is perfectly suited to the production of quasi-optical lenses for the $\mathrm{THz}$ range, where wavelengths are higher than $1 \mathrm{~mm}$.

Achieving good quality of lenses by FDM 3D printing is not as easy as one could expect. The process depends not only on the 3D model but also on various settings and environmental factors related to the machine itself, its properties and technical condition. Print quality can be significantly improved by knowing how a 3D printer works.

FDM is a process of three-dimensional printing polymeric materials that can be used to produce $\mathrm{THz}$ optics. Among the most well-known are: Acrylonitrile butadiene styrene (ABS), Polyactic acid (PLA), Polypropylene (PP), Nylon, BendLay and High Impact Polystyrene (HIPS). For the development of optical elements, important are the refractive indexes and the absorption coefficients of polymer materials for the required $\mathrm{THz}$ frequency range. All analyzed materials have a frequency-independent refractive index for applications within the range 0.2 to $1.4 \mathrm{THz}$ [9]. The initial test frequency was $500 \mathrm{GHz}$ [9]. HIPS was chosen for manufacturing, because it is an alternative to highly absorbing PLA, ABS, nylon and HDPE, PP materials that can be deformed during printing. The absorption coefficient in these materials is higher as compared to that of HIPS and grows with increasing the radiation frequency from 140 up to $270 \mathrm{GHz}$ (see Table 1). HIPS has the refractive index value $n=1.561$ [9]. The 140 and $270 \mathrm{GHz}$ absorption coefficients were determined as being 0.1 and $0.5 \mathrm{~cm}^{-1}$ for HIPS, respectively [9].

Various types of lenses can be used in $\mathrm{THz}$ vision systems, and one of the promising solutions is to use complex (aspherical) surfaces, which helps to reduce aberrations and losses, as well as to diminish the number of lenses in the system to minimize its weight and manufacturing costs.

We propose cylindrical lenses made using 3D printing for applications requiring one-dimensional shaping of a light source such as a line of detectors.
Table 1. Parameters of some materials for 3D printing at 140 and $270 \mathrm{GHz}$ [9].

\begin{tabular}{|c|c|c|c|c|c|}
\hline 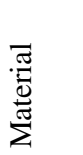 & 离离 & 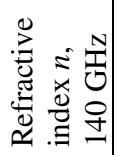 & 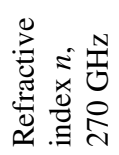 & 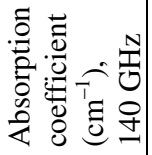 & 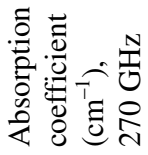 \\
\hline $\mathrm{ABS}$ & 1 & 1.57 & 1.57 & 2 & 3 \\
\hline PLA & 1 & 1.89 & 1.89 & 1 & 6 \\
\hline Nylon & 1 & 1.72 & 1.72 & 0.5 & 4 \\
\hline HIPS & 5 & 1.561 & 1.561 & 0.5 & 0.5 \\
\hline
\end{tabular}

Two types of optical systems for focusing radiation to point or to line were designed and used in systems with a linear array of detectors. Angular dimensions associated with the horn antenna parameters $\left(\omega / 2=14^{\circ}\right)$ and the image quality requirements close to the diffraction limit were taken as initial conditions for the design.

Different diameters of lenses were chosen to focus radiation into different line sizes, namely: $D=50 \mathrm{~mm}$ and $D=80 \mathrm{~mm}$.

The Zemax program was used for calculations of the optical system. This software has a wide range of options to create and optimize optical systems by using ray tracing. In general, Zemax operates in the geometrical optics approximation, and diffraction effects are calculated in a simple approximation or with a limited number of optical surfaces. The focal length and conic constant $k$ were fitted using the least squares method after modeling the optical system in Zemax to maximize a signal power from the source to detector.

The longitudinal coordinate $z$ ('arrow deflection' of surface) was considered as

$$
z=\frac{c \cdot r^{2}}{1+\sqrt{\left(1-(1+k) \cdot c^{2} \cdot r^{2}\right)}},
$$

where $r$ is the distance from the main lens axis, $c-$ inverse radius of curvature of the opposite surface, $k-$ conic constant, which is equal to the square of the eccentricity taken with a minus sign.

The following parameters were obtained: curvature radius of front $(+)$ and rear $(-)$ surfaces was $1 / c=35 \mathrm{~mm}$, conic constant was $k=-2.06$ (hyperboloid second order surface), focal length $-f=80 \mathrm{~mm}$ for the lens with $D=$ $50 \mathrm{~mm}$; curvature radius of front $(+)$ and rear (-) surfaces $-1 / c=65 \mathrm{~mm}$; conic constant $-k=-2.6$ (second order surface - hyperboloid), focal length $-f=125 \mathrm{~mm}$ for the lens with $D=80 \mathrm{~mm}$.

The point spread function (PSF) and full-width at half-maximum (FWHM) are often used to estimate optical system quality.

The settings can help to start trying to improve the quality of $3 \mathrm{D}$ printed parts. It is necessary to consider that different brands and colors of filaments can also differ in print settings to get the same appearance. Also, various printers can be slightly different, and how they are maintained can have an effect on quality.

The printer Creator Flashforge Pro have been used for making lenses from HIPS, thread by MonoFilament 
Table 2. The optimal setting parameters for printing polystyrene lenses.

\begin{tabular}{|l|r|} 
Temperature of table surface, ${ }^{\circ} \mathrm{C}$ & 110 \\
\hline Temperature of nozzle, ${ }^{\circ} \mathrm{C}$ & 230 \\
\hline Height of the layers, $\mu \mathrm{m}$ & 100 \\
\hline Print speed, $\mathrm{mm} / \mathrm{min}$ & 2100 \\
\hline Plastic filling, $\%$ & 100 \\
\hline Material blowing & no \\
\hline
\end{tabular}
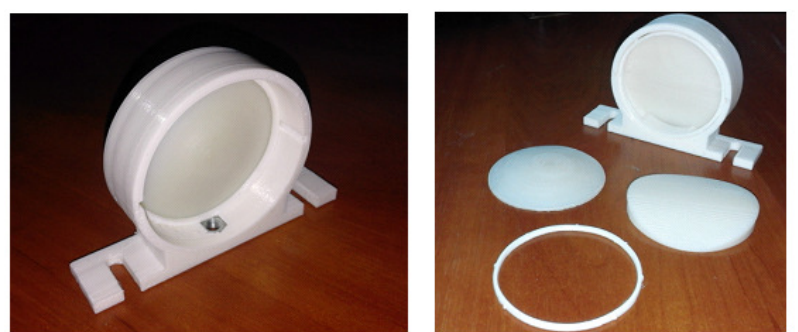

Fig. 1. Lenses and fastenings manufactured on the 3D printer.

with the diameter $1.75 \mathrm{~mm}$. We have collected some settings based on our experience, namely: temperature of table surface and nozzle, the height of the layers, print speed and others (see Table 2).

We have passed complete cycle creation of $\mathrm{THz}$ lenses including: calculation, 3D simulation to 3D fabrication in one workplace. This approach allows significant reduction of the development time of $\mathrm{THz}$ lenses and minimizes their cost.

The printing process of lenses and fastening took approximately three hours. The manufactured parts are shown in Fig. 1.

\section{Testing the THz lenses}

Fig. 2 shows an experimental setup for evaluating quality of the lenses designed and manufactured.

The THz source based on the IMPATT diode with a conical horn antenna was used as the emitter generating continuous, linearly polarized, essentially monochromatic radiation with the power $P \approx 20 \mathrm{~mW}$ at $f=140 \mathrm{GHz}$. The conical antenna has the following parameters: frequency range is $v \approx 110 \ldots 170 \mathrm{GHz}$, gain is $\geq 20 \mathrm{dBi}$, waveguide type is rectangular, waveguide size is WR-6, aperture diameter is $27 \mathrm{~mm}$, horn length is $38 \mathrm{~mm}$.
Tunable BWO source operating within the frequency range $f=128 \ldots 152 \mathrm{GHz}$ and radiation power $P \sim 4 \ldots 10 \mathrm{~mW}$ was also used. At $270 \mathrm{GHz}$, the radiation power from clinotron source was $P \approx 140 \mathrm{~mW}$, gain was $\geq 20 \mathrm{dBi}$, the conical antenna aperture diameter was $25 \mathrm{~mm}$, horn length was $45 \mathrm{~mm}$. In all these sources, horn antennas to transmit $\mathrm{THz}$ power in free space were used.

The pair of lenses focuses the light into point or line (at detector linear array). This scheme was chosen as the intermediate one for simulating illumination of the object in a spot or line followed by focusing on one or line receivers.

To estimate quality of the lenses, the relative power distribution from sources was mapped by $\mathrm{HgCdTe}$ or pyroelectric detectors [10] through the motorized threecoordinate stages displacement (Fig. 1). The output electric signal of detectors was measured using the lockin amplifier Stanford SR 830. The dynamic range of the system was about $60 \mathrm{~dB}$.

In Figs 3 and 4, we show the XY cross section (at $z$ - the focal plane of the lenses) of the 3D the PSF of the lenses for focusing radiation to point and to line, with $f=$ $125 \mathrm{~mm}, a=80 \mathrm{~mm}$. We show comparison of the computer modeling $(c, d)$ and the experimental results $(a, b)$ the PSF fit through the maximum in $x$ - and $y$ direction and photographs of the lenses. The FWHM is stated in Table 3.

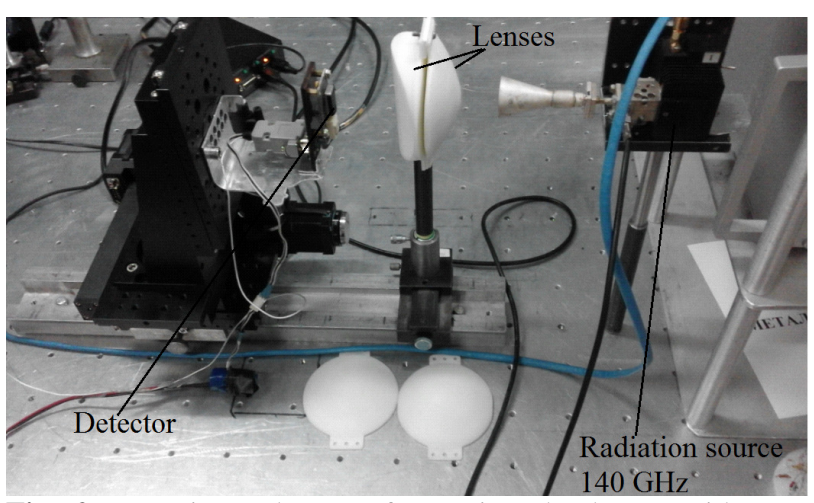

Fig. 2. Experimental setup for testing the lenses with $\mathrm{THz}$ source based on IMPATT diode $(140 \mathrm{GHz})$.

Table 3. The full-width at half-maximum of the collimated and focused beam for different type of lenses at frequencies 140 and $270 \mathrm{GHz}$ are shown. Presents the focusing properties of the lenses and compares with calculated results of the beam diameters, calculated using a Gaussian beam. Both lenses focus properties like expected and showing the dimensions of the spots close to the diffraction limit.

\begin{tabular}{|c|c|c|c|}
\hline \multicolumn{4}{|c|}{ A system from two axially symmetric lenses with the aspherical surfaces, $\varnothing=80 \mathrm{~mm}, f=125 \mathrm{~mm}$} \\
\hline \multicolumn{2}{|c|}{$140 \mathrm{GHz}$} & \multicolumn{2}{|c|}{$270 \mathrm{GHz}$} \\
\hline Calculated FWHM, $x / y, \mathrm{~mm}$ & $\begin{array}{l}\text { Measured FWHM, } \\
x / y, \mathrm{~mm}\end{array}$ & $\begin{array}{l}\text { Calculated FWHM, } \\
x / y, \mathrm{~mm}\end{array}$ & $\begin{array}{l}\text { Measured FWHM, } \\
x / y, \mathrm{~mm}\end{array}$ \\
\hline $4.5 / 4.5$ & 4.5/4.4 & $2 / 2$ & $2.5 / 2.5$ \\
\hline \multicolumn{4}{|c|}{$\begin{array}{l}\text { A system from one axially symmetric lens and one asymmetrical (cylindrical) lens with the aspherical surfaces, } \varnothing=80 \mathrm{~mm} \text {, } \\
\qquad f=125 \mathrm{~mm}\end{array}$} \\
\hline \multicolumn{2}{|c|}{$140 \mathrm{GHz}$} & \multicolumn{2}{|c|}{$270 \mathrm{GHz}$} \\
\hline Calculated FWHM, $x / y, \mathrm{~mm}$ & $\begin{array}{l}\text { Measured FWHM, } \\
x / y, \mathrm{~mm}\end{array}$ & $\begin{array}{l}\text { Calculated FWHM, } \\
x / y, \mathrm{~mm}\end{array}$ & $\begin{array}{l}\text { Measured FWHM, } \\
x / y, \mathrm{~mm}\end{array}$ \\
\hline $\begin{array}{l}\text { 4.5/ image is a line equal to } \\
\text { the lens diameter }\end{array}$ & $4.5 / 10.5$ & $\begin{array}{l}2.5 / \text { image is a line equal to the } \\
\text { lens diameter }\end{array}$ & $2.5 / 5$ \\
\hline
\end{tabular}



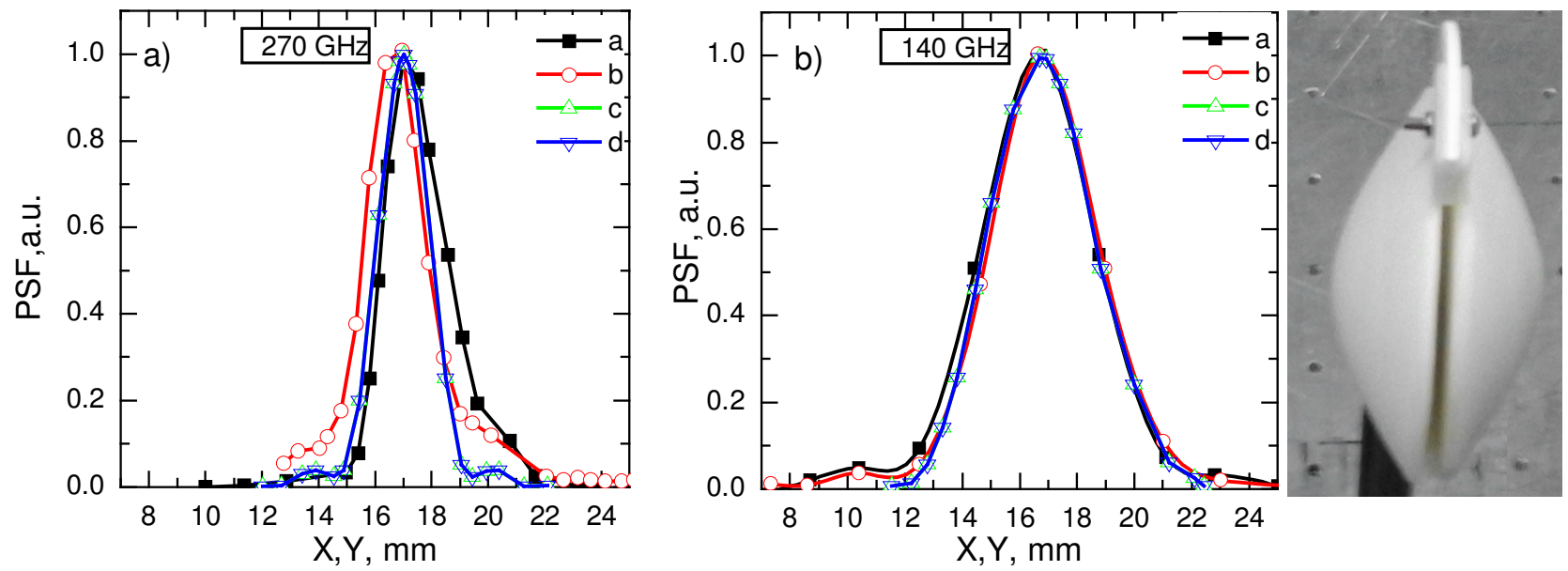

Fig. 3. The system from two axially symmetric lenses with the aspherical surfaces with focal length of $125 \mathrm{~mm}$ and diameter of $80 \mathrm{~mm}$. The comparison of the computer modeling $(c, d)$ and the experimental results $(a, b)$ the PSF fit through the maximum in $x$ and $y$-direction and photographs of the lenses. The full-width at half-maximum is stated in Table 3.
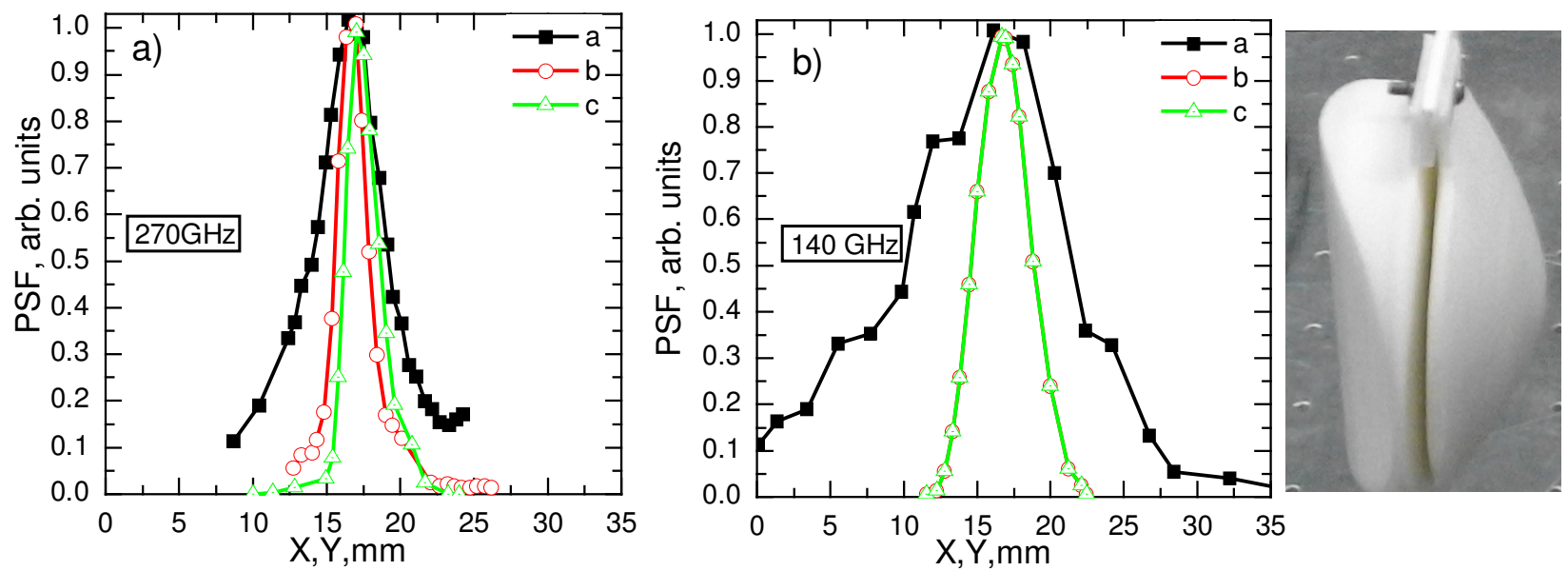

Fig. 4. The system from one axially symmetric lens and one asymmetrical (cylindrical) lens with the aspherical surfaces with focal length of $125 \mathrm{~mm}$ and diameter of $80 \mathrm{~mm}$. The comparison of the computer modeling $(c)$ and the experimental results $(a, b)$ the PSF fit through the maximum in $x$ - and $y$-direction and photographs of the lenses. The full-width at half-maximum is stated in Table 3.

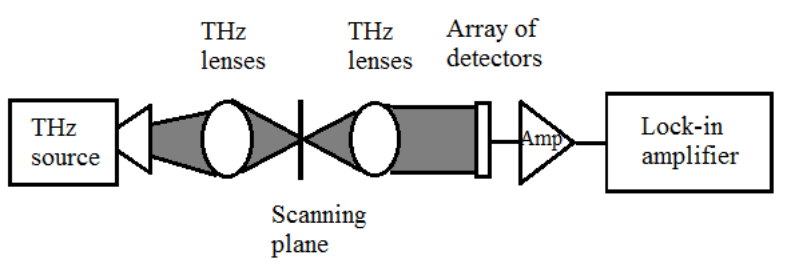

Fig. 5. The block diagram of the optical setup for $X Y Z$ scanning of the objects.

The focusing properties are as expected and show spot sizes that are close to the diffraction limit for the system from two axially symmetric lenses. The results of calculations and measurements differ at $Y$ axis due to the source Gaussian radiation, not Lambertian for the system from one axially symmetric lens and one asymmetrical (cylindrical) lens.

The sizes of the FWHM at $140 \mathrm{GHz}$ are bigger than those at $270 \mathrm{GHz}$ almost twice. That corresponds to the theory that for the same aperture size of the element the spot size depends directly proportional to the frequency.

The THz images were taken using the setup shown in Fig. 5. A narrow parallel beam from the IMPATT diode at $140 \mathrm{GHz}$ was formed using combination lenses. The first pair of lenses from one axially symmetric lens and one asymmetrical (cylindrical) lens focuses the light into the line on the object; the second from two axially symmetric lenses focused it to the 32 elements $\mathrm{THz}$ array detectors (silicon field effect transistors). The detector voltage signal was collected using a preamplifier (Amp) and a lock-in amplifier.

Fig. 6 shows the image of objects obtained using the printed lenses from HIPS material in the $\mathrm{THz}(32$ elements $\mathrm{THz}$ array of total 80 element array, silicon field-effect transistors, pitch $1 \mathrm{~mm}$ ) and visible ranges.

The image was obtained for hidden objects behind the plastic foam of the thickness $d=1 \mathrm{~cm}$. One can clearly see the different washer, pill, and sugar with sufficient spectral resolution $\sim 4.5 \mathrm{~mm}$. 


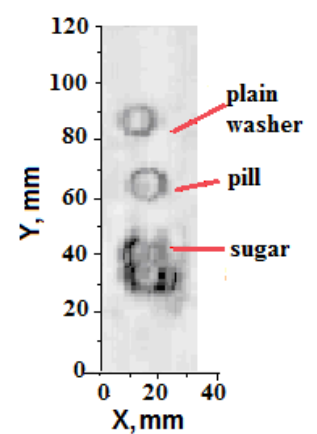

a)

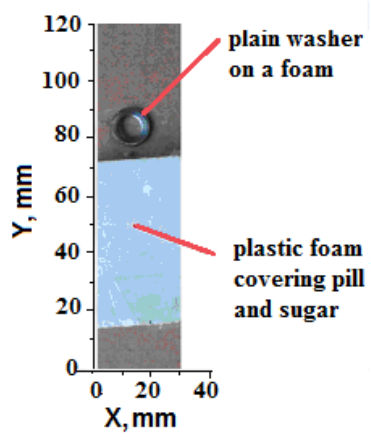

b)
Fig. 6. a) Washer, pill, and sugar that are hidden behind plastic foam of the thickness $d=1 \mathrm{~cm}, \mathrm{~b}$ ) the same objects in the visible range.

The obtained results confirm the possibility to get high-quality aspherical lenses by using the plastic layering method of $3 \mathrm{D}$ printing with close to simulated parameters such as focal lengths and PSF. A quantitative method of determining the quality of this image is calculation of the FWHM and comparison with the experimental data. In our case, they are $\left(\mathrm{FWHM}_{\text {calc }, x / \text { meas }, x}\right.$ $(140 \mathrm{GHz})=4.5 \mathrm{~mm} / 4.5 \mathrm{~mm}, \quad \mathrm{FWHM}_{\text {calc, }, y / \text { meas }, y}$ $(140 \mathrm{GHz})=4.5 \mathrm{~mm} / 4.4 \mathrm{~mm})$ for axially symmetric lenses, $\varnothing=80 \mathrm{~mm}$, which provides resolution $\sim 4.5 \mathrm{~mm}$. The FWHM is FWHM $_{\text {calc }, x / \text { meas }, x} \quad(140 \mathrm{GHz})=$ $4.5 \mathrm{~mm} / 4.5 \mathrm{~mm}$, FWHM calc, $y /$ meas, $y \quad(140 \mathrm{GHz})=$ $10.5 \mathrm{~mm} /$ (image is a line with the length equal to the half lens diameter) for the one axially symmetric lens and one asymmetrical (cylindrical) lens .

\section{Conclusions}

The possibility of manufacturing relatively good-quality $\mathrm{THz}$ lenses from high impact polystyrene material (HIPS) by 3D printing has been shown. It was proposed to use cylindrical lenses for applications requiring onedimensional shaping of a light source such as a line of detectors. HIPS was chosen for manufacturing $\mathrm{THz}$ lenses, because it has advantages compared to highly absorbing PLA, ABS, nylon and HDPE, PP materials that can also be deformed during printing. Images of "hidden in the visible spectrum" objects were obtained with the use of manufactured lenses of sufficient spectral resolution $\sim 4.5 \mathrm{~mm}$ by using the $140 \mathrm{GHz}$ frequency source. Scanning (with the frame rate $\sim 1 \mathrm{~Hz}$ ) in the direction perpendicular to 32-pixel linear detector array based on silicon field-effect transistors has been carried out.

\section{Acknowledgments}

This paper is partly based on researches supported by Volkswagen Project Application No. A115974 "Optoelectronic and transport phenomena in narrow-gap semiconductor structures for terahertz detection".

\section{References}

1. Kai-Erik Peiponen, J. Axel Zeitler, Makoto Kuwata-Gonokami (Eds.), Terahertz Spectroscopy and Imaging. Springer, 2013.

2. Squires A.D., Constable E., Lewis R.A. 3D printing of aspherical terahertz lenses and diffraction gratings. 39th Intern. Conf. on Infrared, Millimeter, and Terahertz waves (IRMMW-THz), IEEE. P. 12.

3. Rachon M., Liebert K., Siemion A., Bomba J., Sobczyk A., Knap W., Coquillat D., Suszek J., Sypek M. Geometrical aberration suppression for large aperture sub-THz lenses. J. Infrared, Millimeter, and Terahertz Waves. 2017. 38, No. 3. P. 347-355.

4. Gallerano G.P., and Biedron S. Overview of terahertz radiation sources. Proc. 2004 FEL Conf. Triest, Italy, 2004. P. 216-221.

5. Paul D.J. The progress towards terahertz quantum cascade lasers on silicon substrates. Laser Photon. Rev. 2010. 4, No. 5. P. 610-632.

6. Yat Hei Lo, Leonhardt R., Torres V., Pacheco-Pena V., Rodriguez-Ulibarri P., Navarro-Cia M., Beruete M., Sorolla M., Enghet N. Aspheric lenses for terahertz imaging. Opt. Exp. 2008. 16, No. 20. P. 15991-15998.

7. Sizov F., Tsybrii Z., Zabudsky V., Sakhno M., Shevchik-Shekera A., Smoliy M., Dieguez E., Dvoretsky S. Possibility of the detection in IR and $\mathrm{sub} / \mathrm{THz}$ spectral region using MCT thin layer receivers: design of the chip, optical elements and antenna pattern. IEEE MMS 2015. Lecce, Italy, 2015, P. 49-52.

8. Sizov F., Tsybrii Z., Zabudsky V., Golenkov O., Petryiakov V., Dvoretsky S., Michailov N., Shevchik-Shekera A., Lysiuk I., Dieguez E. Mercury-cadmium-telluride thin layers as subterahertz and infrared detectors. Opt. Eng. 2015. 54, No. 12. P. 127102-1-127102-8.

9. Busch S., Weidenbach M., Fey M., Schafer F., Probst T., and Koch M. Optical properties of 3D printable plastics in the $\mathrm{THz}$ regime and their application for 3D printed $\mathrm{THz}$ optics. J. Infrared, Millimeter, Terahertz Waves. 2014. 35. P. 993-997.

10. Sizov F., Zabudsky V., Golenkov A., and ShevchikShekera A. Mm-wave narrow-gap uncooled hotcarrier detectors for active imaging. Opt. Eng. 2013. 52, No. 3, P. 033203. 


\section{Authors and CV}

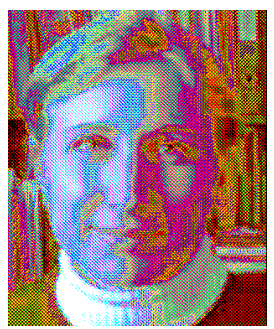

Anna Shevchik-Shekera received the BS and MS degrees in laser and optoelectronic engineering from the National Technical University of Ukraine, Kiev, in 1993 and 1995, respectively. From 2005 to 2008 she was with the Institute of Semiconductor Physics of NASU as a design engineer, where she was involved in the design of thermal imaging cameras. Since 2008, she has been working as a researcher. Her research interests include infrared and millimeter-wave imaging of active vision systems.

V. Lashkaryov Institute of Semiconductor Physics, NAS of Ukraine, Kyiv, Ukraine

E-mail: annashsh82@gmail.com

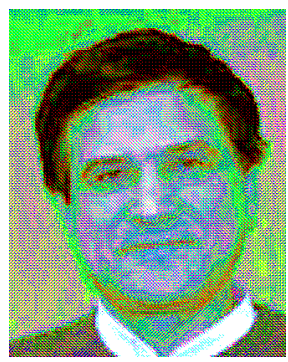

Vyacheslav V. Zabudsky received the $\mathrm{PhD}$ degree in 1997 on the specialty "Physics of semiconductors and dielectrics" from the National Academy of Sciences of Ukraine. His professional activities are in the fields of infrared microphotoelectronics,

semiconductor low-dimension structures, developing of the testing equipment and methods, investigation of $\mathrm{HgCdTe}$ detecting properties, infrared and terahertz detectors and vision systems in these spectral regions. In these areas, he has published more than 50 scientific and technical papers.

V. Lashkaryov Institute of Semiconductor Physics, NAS of Ukraine, Kyiv, Ukraine

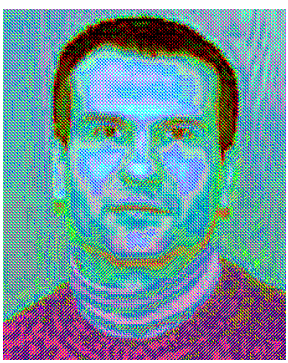

Aleksandr G. Golenkov graduated from the physics department of Kyiv State University (1996), and received his $\mathrm{PhD}$ degree on the specialty "Physics of devices, elements and systems" (2008). His work experience is in the fields of infrared and microwave electronics, semiconductor low-dimension structures, testing equipment and methods, information reading and processing methods, investigation of the infrared (MCT) and terahertz (MOSFET) detectors.

V. Lashkaryov Institute of Semiconductor Physics, NAS of Ukraine, Kyiv, Ukraine

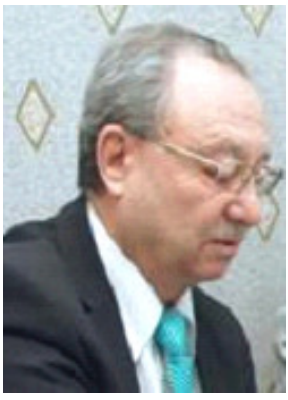

Dvoretskiy Sergey Alekseevich, $\mathrm{PhD}$ from the Institute of Semiconductor Physics of Siberian Branch of Academy of Sciences of Russia, 1975. 19691995, Institute of Semiconductor Physics, Novosibirsk. Junior scientific researcher (1972), senior scientific researcher (1987), leader of group of MBE

II-VI compound (1988), Head of Laboratory (2012), till now. The basic research is the growth II-VI compound including narrow gap $\mathrm{HgCdTe}$ hetero- and nanostructures.

A.V. Rzhanov Institute of Semiconductor Physics, RAS, pr. Lavrentieva, 13, Novosibirsk, Russian Federation 